\title{
Assessment of the number of umbilical cord vessels at the time of nuchal translucency screening
}

\begin{abstract}
Objective: To investigate feasibility of identifying the number of vessels in umbilical cord during nuchal translucency screening.

Methods: We performed a prospective study in patients undergoing nuchal translucency screening at 11.0-13.9 weeks' from 2011-2012. An oblique transverse view of fetal abdomen utilizing Doppler flow and a cross section view of free loop of umbilical cord were obtained. Maternal characteristics, technical aspects of first trimester ultrasound, and number of umbilical cord vessels identified in the second trimester were collected. McNemar's test and kappa analysis were used to compare quantification of umbilical cord vessels in the first vs. second trimesters.

Results: Total of 123 women was included. The number of umbilical cord vessels was successfully visualized in $95.9 \%$ of patients utilizing the bladder view, and $65.0 \%$ of patients on cross sectional cord views. Three-vessel cord was correctly identified in $99.2 \%$ of patients; one case was incorrectly diagnosed with a two two-vessel cord. McNemar's test indicated that overall, first and second trimester ultrasounds have the same propensity to detect umbilical cord vessels $(\mathrm{p}=0.414)$, with moderate agreement $(\mathrm{kappa}=0.226)$
\end{abstract}

Conclusion: Identification of umbilical cord vasculature is possible in the first trimester and can be used as an adjunct to early fetal anatomy evaluation.
Volume I Issue 2 - 2014

\author{
Julia Timofeev,' Marium Holland,' Cecily \\ Clark Ganheart,' Helain J Landy, ${ }^{2}$ Eshetu \\ Tefera, ${ }^{3}$ Rita W Driggers ${ }^{1}$ \\ 'Department of Obstetrics and Gynecology, MedStar \\ Washington Hospital Center, USA \\ ${ }^{2}$ Department of Obstetrics and Gynecology, MedStar \\ Georgetown University Hospital, USA \\ ${ }^{3}$ Department of Biostatistics \& Epidemiology, MedStar Health
} Research Institute, USA

Correspondence: Julia Timofeev, Department of Obstetrics and Gynecology, MedStar Washington Hospital Center, I 10 Irving Street NW, 5B-63, Washington, DC 200I0, USA, Tel 352 219-4408, Fax 202-877-5435, Email juliati@gmail.com

Received: October 27, 2014 | Published: November 13,2014

Keywords: umbilical cord, trimester, fetal, ultrasound, gestational

Abbreviations: NT, nuchal translucency; CRL, crown-rump length; PAPP-A, pregnancy-associated plasma protein A; B-HCG, beta human chorionic gonadotropin; SUA, single umbilical artery; BMI, body mass index; TI, thermal index; MI, mechanical index

\section{Introduction}

Approximately $4 \%$ of pregnancies have one or more major fetal malformations and $2 \%$ are affected by a genetic abnormality. ${ }^{1}$ Screening for fetal anomalies classically has taken place during the second trimester of pregnancy; however, with improving ultrasound technology more fetal malformations are being detected earlier in gestation. Nuchal translucency (NT) thickness is utilized as a screening test in prenatal assessment of risk of aneuploidy in the first trimester. This test is performed between 11.0-13.9 weeks gestational age, corresponding to a crown-rump length (CRL) of $45-84 \mathrm{~mm}$. Alone, or in combination with maternal serum analytes of pregnancyassociated plasma protein A (PAPP-A) and beta human chorionic gonadotropin (B-HCG), NT can detect $64-70 \%$ or $82-87 \%$ of Down syndrome cases, respectively, assuming a $5 \%$ screen positive rate Physicians and sonographers performing NT screening undergo an initial certification process and ongoing quality review to maintain accreditation and ensure consistent results.

Recently, several studies have examined the feasibility of early anatomic survey at the time of NT screening, as organogenesis is mostly complete by this gestational age and early detection of anomalies may be beneficial in directing further diagnostic testing. ${ }^{3-7}$ Currently, evaluation of anatomy during first trimester may include visualization of skull and brain, face, spine, abdomen, bladder and extremities. Fetal echocardiography also has been described at the time of NT screening, being performed with more frequency at earlier gestations..$^{7-9}$

A single umbilical artery (SUA) is present in up to $1 \%$ of live ${ }^{10}$ as an isolated finding or in association with other malformations, most commonly cardiac, genitourinary and skeletal. ${ }^{11,12}$ SUA is higher in multiple gestations as well as in trisomies and has been associated with maternal smoking, diabetes mellitus, epilepsy and hypertension. ${ }^{13}$ Absence of one artery can be related to obliteration of a previously normal artery, fusion of the two arteries, or atrophy of the vessel, and carries an increased risk of adverse fetal outcome. ${ }^{11}$ During standard second trimester anatomic survey, the number of umbilical vessels is determined by visualization of the color Doppler flow of the arteries as they course around the bladder and/or by visualizing the cross section of free-floating loop of umbilical cord. In retrospective review by Hill and colleagues, detection of a two-vessel or three-vessel umbilical cord was best achieved between 17-36 weeks gestation with transverse image of umbilical cord. ${ }^{14}$ The investigators noted that the main reasons for failure to visualize the number of vessels were earlier gestation, maternal body habitus, low amniotic fluid volume, and the position of the umbilical cord within the gestational sac. The rate of visualization of the umbilical cord increased from 15 to 17 weeks ( $74.1 \%$ to $97.6 \%$, respectively) and then remained stable until 36.9 weeks. ${ }^{14}$

Our objectives were to prospectively investigate the feasibility of visualization of number of vessels in the umbilical cord at the time of NT screening between 11.0 and 13.9 weeks gestation, and to determine the agreement of transabdominal sonography (including color Doppler mapping) for evaluation of cord vasculature performed during sonography in the first as compared to the second trimester. 


\section{Methods}

All women 18 years and older, presenting for NT screening to MedStar Washington Hospital Center Perinatal Center or MedStar Georgetown University Hospital Prenatal Diagnostic and Ultrasound Center between 11.0-3.9 weeks' and meeting CRL requirement (45$84 \mathrm{~mm}$ ) were invited to participate in the study. Informed consent was obtained by the physician identifying the patient as a potential candidate for the investigation.

This study was approved by MedStar Health Research Institute IRB with enrollment commencing in March 2011 and concluding in June 2012, and is registered on clinicaltrials.gov (NCT01367132).

Gestational age was established by a certain last menstrual period if available and if correlated with the CRL measurement \pm 5 days. The conception date was used for women who underwent assisted reproductive therapy. If a last menstrual period was not known, gestational age was defined as measured by the earliest sonographic evaluation. CRL measurement was obtained at the beginning of the nuchal translucency screening to confirm gestational age and to ascertain that the fetal CRLmet criteria for the screening parameters of NT.

Enrolled patients then underwent standard NT screening performed by physicians and/or sonographers accredited by either Fetal Medicine Foundation or the Nuchal Translucency Quality Review Program from the Society of Maternal Fetal Medicine in this technique. Full evaluation included transabdominal scanning for the measurement of CRL, fetal heart rate, the sonolucent area in the posterior fetal neck, as well as limited evaluation of the fetal anatomy including: skull and brain (examination for completeness of skull and symmetric choroid plexuses/ventricles), face (fetal profile and presence or absence of nasal bone if certified in nasal-bone screening), spine, thorax (four chamber view of the heart), abdomen (stomach, abdominal wall and umbilical cord insertion site), bladder and extremities (upper and lower). Location of the placenta and placental cord insertion were also noted. All imaging was performed with Voluson E8 Expert (GE Healthcare) or the iU22 ultrasound systems (Phillips). Serum analytes ( $\beta$-HCG and PAPP-A) were collected in accordance with laboratory requirements. The patient was notified of the results of the First Trimester Screening by their providers, once the risk assessment was available from the reference laboratory (PerkinElmer Labs/NTD, Melville, New York).

Upon completion of the standard NT evaluation, an oblique transverse section of the lower fetal abdomen including the fetal bladder was visualized transabdominally. Doppler color flow mapping was utilized to visualize the umbilical arteries as they traverse around both sides of the bladder in continuity with the umbilical cord insertion of the fetus (bladder view). If an artery was seen on both sides of the bladder, the finding was recorded as a three-vessel umbilical cord. If only one artery was seen on either side of the bladder, this was recorded as a two-vessel cord. A cross sectional view of the freefloating umbilical cord was then obtained and recorded as consistent with a three- or two-vessel cord.

The provider assessing the vasculature of the umbilical cord could share the findings with the patient per their discretion. However, the findings were not used as a sole reason for recommending invasive diagnostic testing (e.g. chorionic villus sampling [CVS]), as the accuracy of the detection rate of vessels in the first trimester has not been established and may be significantly limited by early gestational age.

Assessment of number of vessels in the cord was performed as a standard practice at the time of routine second trimester sonogram for anatomic survey (approximately at 18-24 weeks' gestation). The transabdominally obtained sonographic views were the same as described during NT screening, and utilized Doppler color flow mapping to visualize the umbilical arteries as they traverse around the bladder, and to obtain a cross sectional view of the free-floating loop of the umbilical cord. The number of vessels in the umbilical cord was recorded and compared to the determination made during NT screening sonogram.

Maternal demographic information including age, parity, last menstrual period, gestational age at presentation, use of tobacco, body mass index (BMI) and chronic medical conditions (hypertension, diabetes, neurologic or thrombophilic disorders) were obtained at the time of enrollment.

First-trimester sonogram data was extracted from ultrasound report including CRL and corresponding gestational age at the time of NT screening, number of vessels of umbilical cord, and any other noted findings. Second-trimester sonogram data was obtained from ultrasound report including number of vessels of the umbilical cord and any noted fetal anomalies. Information collected from the reports was verified by the review of all sonographic images by two investigators (J.T. and C.C-G.).

The number of umbilical cord vessels determined during first and second trimester ultrasound scans also was compared to the description of the number of vessels noted at the time of birth in the delivery report and/or placental pathology report if available.

The primary study outcome was the visualization of the number of vessels in the umbilical cord between 11.0-13.9 weeks' gestation as compared to standard technique of determining vasculature of the cord in the second trimester. Descriptive statistics of the demographic data were calculated. Sensitivity, specificity, positive and negative predictive values were determined for sonographically acquired determination of the vasculature of the cord in the first and second trimesters and comparisons were made utilizing the McNemar's test and kappa analysis for the detection rate of umbilical cord vasculature in the first vs. the second trimester ultrasound.

\section{Results}

A total of 123 women granted consent to participate in the study (Figure 1). Maternal characteristics are presented in Table 1. Technical aspects of first trimester evaluation are presented in Table 2. All NT evaluations were performed using transabdominal ultrasound; there were no cases using transvaginal scanning. During the NT screening, the mean gestational age was $12.6 \pm 0.6$ weeks, and CRL was $63.2 \pm 8.4$ $\mathrm{mm}$. Thermal index (TIb) and mechanical index (MI) were $0.5 \pm 0.2$ and $1.2 \pm 0.1$, respectively, with the mean length of exposure to Doppler evaluation of 62 seconds.

The number of vessels in the umbilical cord was successfully visualized in $118 / 123$ patients $(95.9 \%)$ on bladder view, and $80 / 123$ $(65.0 \%)$ cross sectional views. The detection rate of umbilical cord vessels increased with increasing gestational age for both bladder and cross section views, reaching $100.0 \%$ of cases on bladder view and $73.3 \%$ of cases on cross sectional views by 13.0 to 13.9 weeks (Table 
3). Detection rates utilizing bladder and cross sectional views were stable over maternal body mass index categories (Table 4). Overall comparison of bladder and cross sectional views of the umbilical cord in the first trimester was comparable to visualization in the second trimester $(\mathrm{p}=0.414)$ with moderate agreement (kappa $=0.226)$.

Table 1 Demographics

\begin{tabular}{|c|c|}
\hline & $n=\mid 23$ \\
\hline Age $(y r) m e a n \pm S D$ & $27.7 \pm 6.3$ \\
\hline Gravidity median (IQR) & $2(1,4)$ \\
\hline \multicolumn{2}{|l|}{ Parity, n (\%) } \\
\hline Nulliparous & $56(46.7)$ \\
\hline Multiparous & $64(54.3)$ \\
\hline \multicolumn{2}{|l|}{ BMI $\left(\mathrm{kg} / \mathrm{m}^{2}\right)$} \\
\hline mean $\pm S D$ & $30.5 \pm 6.3$ \\
\hline median (IQR) & $29.4(26.8,33.6)$ \\
\hline Tobacco use, n (\%) & $6(5.2)$ \\
\hline \multicolumn{2}{|c|}{ Medical comorbidities, n (\%) } \\
\hline Chronic hypertension & $6(5.2)$ \\
\hline Preexisting diabetes & $2(1.7)$ \\
\hline Seizure disorder & --- \\
\hline Thrombophilia & --- \\
\hline \multicolumn{2}{|l|}{ Gestational age (weeks) } \\
\hline mean $\pm S D$ & $12.6 \pm 0.6$ \\
\hline median (IQR) & $12.4(12.1,12.9)$ \\
\hline \multicolumn{2}{|l|}{ Crown rump length (mm) } \\
\hline mean $\pm S D$ & $63.2 \pm 8.4$ \\
\hline median (IQR) & $63.2(57.1,67.8)$ \\
\hline
\end{tabular}

BMI, body mass index; SD, standard deviation; IQR, interquartile range

Table 3 Visualization of umbilical cord vessels by gestational age in the first trimester

\begin{tabular}{llllll}
\hline \multirow{2}{*}{ GA (wks) } & \multirow{2}{*}{ Number of cases $\mathbf{n}(\%)$} & \multicolumn{2}{l}{ Bladder view } & \multicolumn{2}{c}{ Cross section view } \\
\cline { 3 - 6 } & Visualized & Not visualized & \multicolumn{2}{l}{ Visualized } & Not visualized \\
$\mathbf{n}(\%)$ & $\mathbf{n}(\%)$ & $\mathbf{n}(\%)$ \\
\hline $11.0-11.9$ & $17(13.8)$ & $16(94.1)$ & $1(5.9)$ & $10(58.8)$ & $7(41.2)$ \\
$12.0-12.9$ & $76(61.8)$ & $72(94.7)$ & $4(5.3)$ & $48(63.2)$ & $28(36.8)$ \\
$13.0-13.9$ & $30(24.4)$ & $30(100.0)$ & $0(0.0)$ & $22(73.3)$ & $8(26.7)$ \\
$11.0-13.9$ & 123 & & & & \\
\end{tabular}

GA, gestational age 


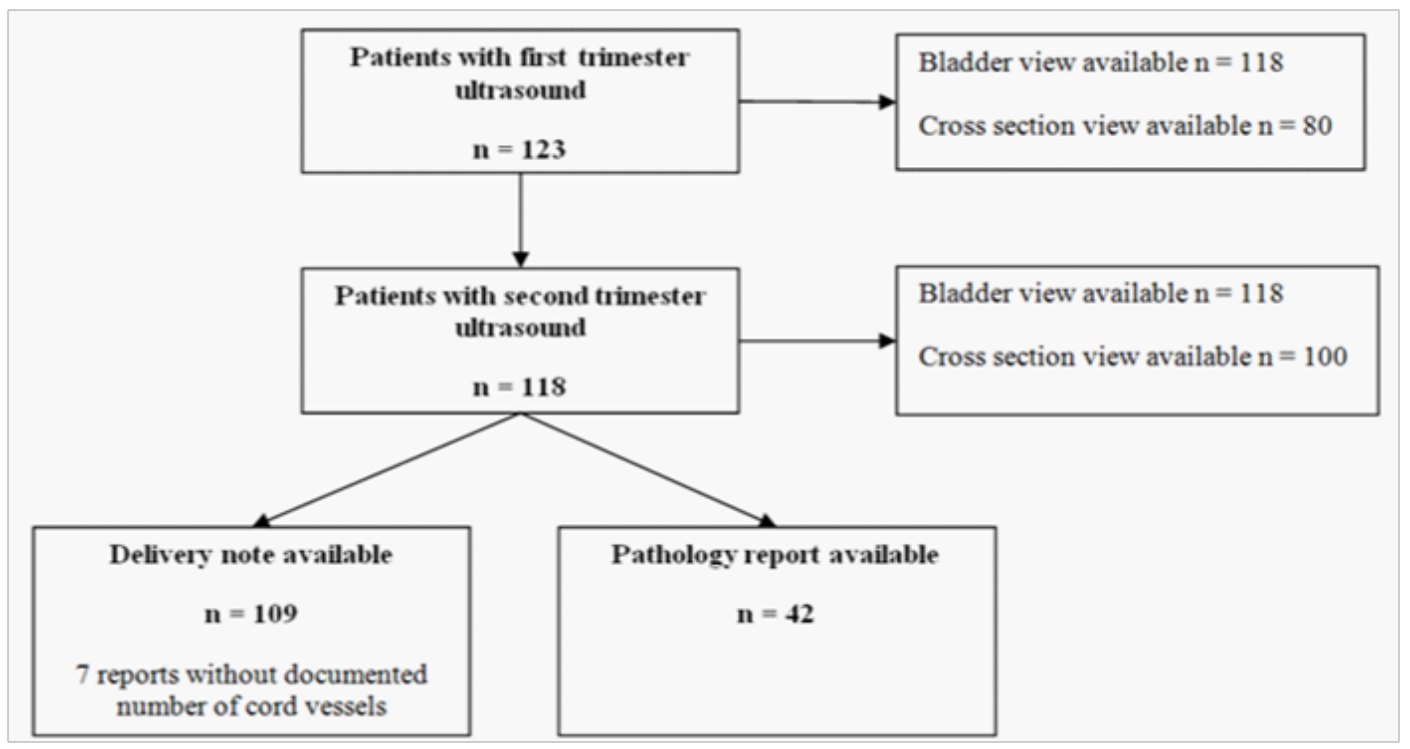

Figure I Patient selection diagram.

Table 4 Visualization of umbilical cord vessels by gestational age in the first trimester by maternal body mass index

\begin{tabular}{llllll}
\hline & & \multicolumn{2}{c}{ Bladder view } & \multicolumn{2}{c}{ Cross section view } \\
\cline { 3 - 6 } BMI $\left(\mathbf{k g} / \mathbf{m}^{2}\right)$ & Number of cases* $\mathbf{n}(\%)$ & Visualized & Not visualized & Visualized & Not visualized \\
& & $\mathbf{n}(\%)$ & $\mathbf{n}(\%)$ & $\mathbf{n}(\%)$ & $\mathbf{n}(\%)$ \\
\hline $18.5-24.9$ & $18(15.8)$ & $18(100.0)$ & $0(0.0)$ & $11(61.1)$ & $7(3.9)$ \\
$25.0-29.9$ & $46(40.4)$ & $44(95.7)$ & $2(4.3)$ & $31(67.4)$ & $15(32.6)$ \\
$\geq 30.0$ & $50(43.9)$ & $48(96.0)$ & $2(4.0)$ & $33(66.0)$ & $17(34.0)$ \\
\hline
\end{tabular}

*Nine subjects had missing body mass index (BMI) information, effective $\mathrm{n}=\mathrm{II} 4$

Table 5 Detection of umbilical cord vessels in the first and second trimester as compared to after delivery evaluation

\begin{tabular}{|c|c|c|c|c|c|c|}
\hline \multirow{2}{*}{$\begin{array}{l}\text { Number of } \\
\text { vessels }\end{array}$} & \multicolumn{2}{|c|}{ First trimester $n=\mid 23$} & \multicolumn{2}{|c|}{ Second trimester $n=\mid$ | 8} & \multirow{2}{*}{$\begin{array}{l}\text { Delivery note } \\
n=109\end{array}$} & \multirow{2}{*}{$\begin{array}{l}\text { Placental } \\
\text { pathology } \\
n=42\end{array}$} \\
\hline & Bladder view & Cross section view & Bladder view & Cross section view & & \\
\hline 3 vessels & II 7 (95.I) & $79(64.2)$ & $118(100.0)$ & $100(84.7)$ & $102(93.6)$ & $42(100.0)$ \\
\hline 2 vessels & $\mathrm{I}(0.8)$ & $\mathrm{I}(0.8)$ & 0 & 0 & 0 & 0 \\
\hline Not visualized & $5(4.1)$ & $43(35.0)$ & 0 & $18(15.3)$ & $7(6.4)$ & N/A \\
\hline
\end{tabular}

Table 6 Sensitivity and specificity of first trimester ultrasound in umbilical cord vessel assessment vs. second trimester

\begin{tabular}{llll}
\hline & Bladder view & Cross section & Overall \\
\hline Sensitivity & $96.60 \%$ & $64.00 \%$ & $98.30 \%$ \\
Specificity & $0 \%$ & $27.80 \%$ & $20.00 \%$ \\
PPV & $100.00 \%$ & $83.10 \%$ & $96.70 \%$ \\
NPV & $0 \%$ & $12.20 \%$ & $33.30 \%$ \\
\hline
\end{tabular}

PPV, positive predictive value; NPV, negative predictive value 


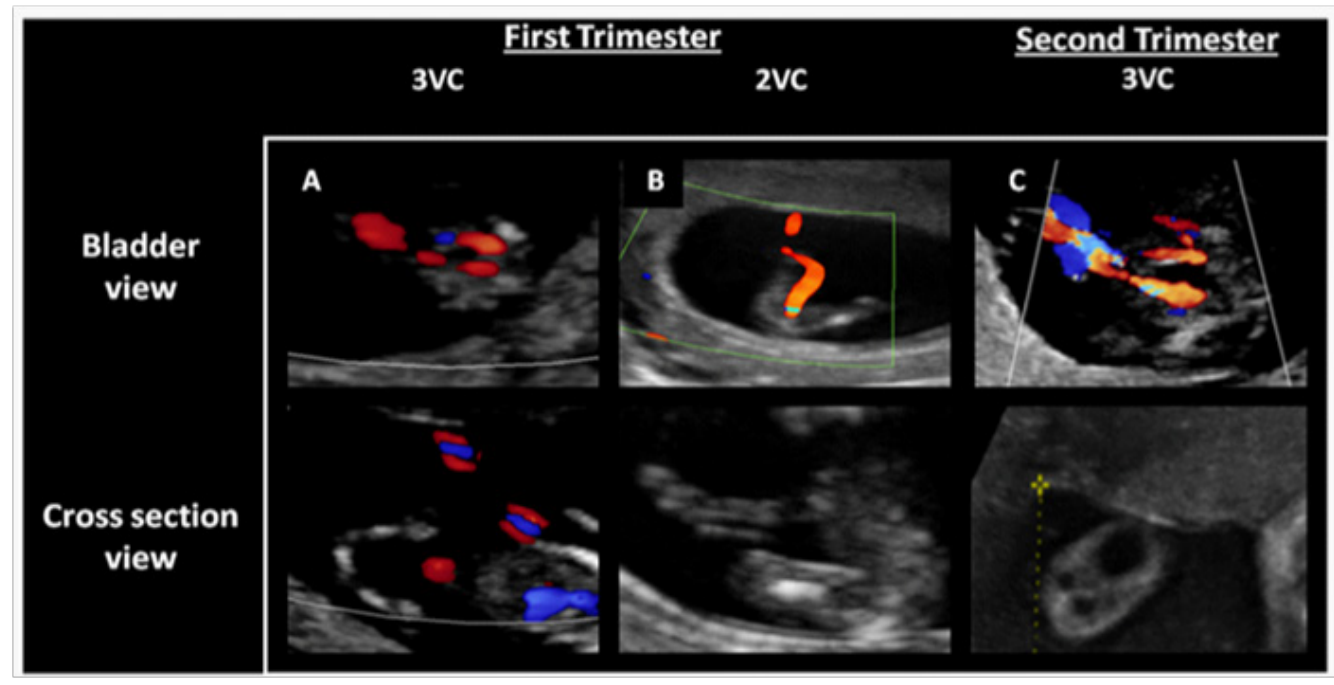

Figure 2 Bladder and cross sectional views of the umbilical cord in the first and second trimesters. A: Normal appearance of the three-vessel cord during the first trimester; B:Appearance of the umbilical cord during the first trimester ultrasound in the misclassified case; C:Appearance of the umbilical cord during the second trimester ultrasound in the misclassified case. 3VC-Three-Vessel Cord; 2VC-Two-Vessel Cord.

\section{Discussion}

It has been estimated, that at 11.0-13.9 week ultrasound, SUA is found in $3.3 \%$ of karyotypically normal fetuses and in $77.8 \%, 11.4 \%$, and $9.5 \%$ of fetuses with Trisomy 18,21 , and other chromosomal abnormalities, respectively. ${ }^{15}$ In the second and third trimesters, the rate of chromosomal abnormalities can be as high as $50 \%$ when SUA is seen in combination with congenital anomalies. ${ }^{16}$

Our objective was to investigate the feasibility of identification of the umbilical cord vasculature during the first trimester given that SUA can be associated with other anomalies and genetic conditions. Our study was successful in demonstrating that the umbilical cord vasculature could be visualized in the first trimester in the majority of patients $(95.9 \%$ of patients on bladder view and $65.0 \%$ of patients on cross sectional views). Successful visualization increased with advancing gestational age after 13.0 weeks' gestation $(100.0 \%$ for bladder view and $73.3 \%$ for cross sectional views). The one misclassified case in our study (a three-vessel cord identified as a twovessel cord) relied on both the bladder and cross sectional transverse views of the cord, and neither approach was helpful in correctly delineating the number of vessels. In this particular case, it is possible that ascertainment bias could have played a role given that a cystic hygroma also was present at the time of the study. Although study by Hill et al. ${ }^{14}$ in the second trimester recommended assessment of umbilical cord vessels in transverse plane of free-floating loop of the cord, we found that first trimester visualization using this technique was more difficult than the bladder view.

Other limitations of the study are the relatively small sample size and limited delivery and pathology report information. The main goals, however, to determine visualization of umbilical cord vasculature in the first trimester and to compare to the second trimester ultrasound assessment, were accomplished with high accuracy. A larger prospective study is needed to evaluate the sensitivity, specificity, and positive and negative predictive values of first trimester transabdominal sonography in detection of a two-vessel umbilical cord.
The accuracy of detection of umbilical cord vessels could only be evaluated in relation to detection of a three-vessel cord as there were no cases of a two-vessel cord noted in our cohort. This was accomplished successfully in $99.2 \%$ of cases.

Diagnostic ultrasound has been utilized in obstetrics for many decades and has a great record of safety with no clear adverse effects in humans provided that safety guidelines are followed. There are bioeffects that are inherent to any technology and, for use of ultrasound, as a form of energy; concerns are focused on the thermal index (TIs - soft tissue only; TIb-in the presence of bone, indicator of potential temperature increase) and the mechanical index (MI, indicator of potential cavitational effects). Conventional ultrasound evaluation is performed in B-mode (gray scale) which is low power, with an associated TI of $0.2 \pm 0.1 .^{17}$ The addition of Doppler can, however, create outputs that are much higher (especially in case of pulsed Doppler), and the main concern would then appear to be that of heating (reflected by the TI of $0.8 \pm 0.1$ for color Doppler and $1.5 \pm 0.1$ in pulsed Doppler studies). ${ }^{18}$ Theoretically, effects can be enhanced even further by performance of transvaginal Doppler studies.

Guidelines exist on maximum scanning times in obstetric ultrasound evaluation, based on values of TI. ${ }^{19,20}$ For example, the scan should be limited to $<15$ minutes at TI $1.5-2$, while for TI $0-0.7$, scanning time is theoretically unlimited, but is still guided by the ALARA principle (as low as reasonably achievable) in order to achieve a diagnostic image. It is important to note that there are no documented iatrogenic effects on the fetus of Doppler ultrasound that is performed at appropriate levels of power. Rembouskos et al., ${ }^{15}$ found that determination of the number of vessels in the umbilical cord during first trimester, added approximately 1 minute to the ultrasound examination, and thus provided only a minimal amount of exposure of the fetus to color flow Doppler (the lowest power output of all Doppler modalities available). NT examination is carried out at the time when organogenesis is mostly complete, and no transvaginal Doppler studies were utilized in this investigational protocol. In our study, median Doppler exposure time was 53 seconds, ranging 
from 30-90 seconds, which is similar to exposure time reported by Rembouskos and colleagues. ${ }^{15} \mathrm{TIb}$ and MI were in the acceptable safety range of ultrasound evaluation.

The finding of a two-vessel cord during the second trimester should prompt close evaluation for other anomalies, and may lead to recommendation for invasive diagnostic testing. Similarly, evaluation of number of vessels in the umbilical cord during the first trimester may be an informative adjunct to the limited anatomic evaluation performed at the early gestational age, allowing for additional targeted evaluation or diagnostic testing to be conducted at an earlier gestational age.

\section{Acknowledgments}

We thank Yunhui Zhang, RDMS, and Shay Bahmanian, RDMS, for assistance with recruitment in the study.

\section{Conflict of interest}

The authors declare that there is no conflict of interest.

\section{References}

1. Cuckle H, Nanchahal K, Wald N. Birth prevalence of down's syndrome in England and wales. Prenat Diagn. 1991;11(1):29-34.

2. ACOG Committee on Practice Bulletins. ACOG practice bulletin no. 77: Screening for fetal chromosomal abnormalities. Obstet Gynecol. 2007;109(1):217-227.

3. Souka AP, Nicolaides KH. Diagnosis of fetal abnormalities at the 10-14week scan. Ultrasound Obstet Gynecol. 1997;10(6):429-442.

4. Dugoff L. Ultrasound diagnosis of structural abnormalities in the first trimester. Prenat Diagn. 2002;22(4):316-320.

5. Whitlow BJ, Chatzipapas IK, Lazanakis ML, et al. The value of sonography in early pregnancy for the detection of fetal abnormalities in an unselected population. Br J Obstet Gynaecol. 1999;106(9):929-936.

6. Economides DL, Whitlow BJ, Braithwaite JM. Ultrasonography in the detection of fetal anomalies in early pregnancy. Br J Obstet Gynaecol. 1999;106(6):516-523.

7. Becker R, Wegner RD. Detailed screening for fetal anomalies and cardiac defects at the 11-13-week scan. Ultrasound Obstet Gynecol. 2006;27(6):613-638.
8. Abu-Rustum RS, Daou L, Abu-Rustum SE. Role of ultrasonography in early gestation in the diagnosis of congenital heart defects. J Ultrasound Med. 2010;29(5):817-821.

9. Lombardi CM, Bellotti M, Fesslova V, et al. Fetal echocardiography at the time of the nuchal translucency scan. Ultrasound Obstet Gynecol. 2007;29(3):249-57.

10. Heifetz SA. The umbilical cord: Obstetrically important lesions. Clin Obstet Gynecol. 1996;39(3):571-587.

11. Murphy-Kaulbeck L, Dodds L, Joseph KS, et al. Single umbilical artery risk factors and pregnancy outcomes. Obstet Gynecol. 2010;116(4):843850 .

12. Parilla BV, Tamura RK, MacGregor SN, et al. The clinical significance of a single umbilical artery as an isolated finding on prenatal ultrasound. Obstet Gynecol. 1995;85(4):570-572.

13. Heifetz SA. Single umbilical artery. A statistical analysis of 237 autopsy cases and review of the literature. Perspect Pediatr Pathol. 1984;8(4):345378 .

14. Hill LM, Wibner D, Gonzales P, et al. Validity of transabdominal sonography in the detection of a two-vessel umbilical cord. Obstet Gynecol. 2001;98(5 Pt 1):837-842.

15. Rembouskos G, Cicero S, Longo D, et al. Single umbilical artery at 1114 weeks' gestation: Relation to chromosomal defects. Ultrasound Obstet Gynecol. 2003;22(6):567-570.

16. Nyberg DA, Mahony BS, Luthy D, et al. Single umbilical artery. Prenatal detection of concurrent anomalies. J Ultrasound Med. 1991;10(5):247253.

17. Sheiner E, Shoham-Vardi I, Hussey MJ, et al. First-trimester sonography: Is the fetus exposed to high levels of acoustic energy? J Clin Ultrasound. 2007;35(5):245-249.

18. Sheiner E, Shoham-Vardi I, Pombar X, et al. An increased thermal index can be achieved when performing Doppler studies in obstetric sonography. J Ultrasound Med. 2007;26(1):71-76.

19. American Institute of Ultrasound in Medicine. AIUM practice guideline for the performance of obstetric ultrasound examinations. J Ultrasound Med. 2010;29(1):157-166.

20. Barnett SB, Maulik D, International Perinatal Doppler Society. Guidelines and recommendations for safe use of Doppler ultrasound in perinatal applications. J Matern Fetal Med. 2001;10(2):75-84. 